\title{
Rare presentation of a benign smooth muscle tumour of venous wall
}

\author{
Tomasz Brzezinski, Marcin Oseka, Maciej Skorski \\ Department of General and Endocrine Surgery, Warsaw Medical University, Poland
}

\begin{abstract}
Deep veins thrombosis is commonly encountered in clinical practice. The reasons and risk factors are well known. Unusual conditions leading to complete obstruction of larger veins are rarely anticipated.

A 34-years-old woman was treated in our hospital because of segmental thrombosis of her right common femoral vein.

Color duplex Doppler revealed a solitary thrombus 5 centimeters in lengths narrowing it by 80-90\%. During emergency operation a small tumour in the orifice of greater saphenous vein was found with adhering thrombus.

Segmental thrombosis of large vein can be connected with an occult underlying pathology. Color duplex Doppler alone does not allow complete evaluation of such problems.
\end{abstract}

Key words: tumour, veins, solitary thrombus

Acta Angiol 2017; 23, I: 17-19

\section{Introduction}

Benign tumours of veins are rare clinical entities. Leiomyomas may arise from the smooth muscles of a blood vessel wall .Most frequently they are encountered in the uterus [I]. When they present as palpable mass it is easier to diagnose them but small tumours can easily be overlooked and diagnosis is made during surgery.

\section{Case study}

The patient is a 34-year-old woman who was admitted to the hospital because of a non painful swelling of her right thigh which she had noted in the last two weeks. No other symptoms were present. The medical history was non-specific. She had delivered two kids in the past without complications and she had never complained of vericose veins. There had been no history of oral contraceptives and other risk factors for thrombophlebitis. Imaging with color duplex Doppler revealed a solitary $5 \mathrm{~cm}$ long thrombus in the right common femoral vein, narrowing it by $80-90 \%$. Other deep veins and superficial ones were patent. No inguinal adenopathy or pathological mass was found in this site. The patient was scheduled for emergency operation because of the apprehension that described thrombus might have migrated to the lungs. During surgery femoral blood vessels were dissected. The common femoral vein contained a thrombus located distal to the sapheno-femoral junction, $5 \mathrm{~cm}$ in length. The femoral vein under inguinal ligament was soft and patent. Proximal part of greater saphenous vein presented as an oval distention measuring $1,5 \times 1 \mathrm{~cm}$. covered with normal adventitia, resembling a small tumour. It was quite solid on palpation. The cross section of the lesion revealed a whitish solid mass that could be a tumour or an unusually shaped old thrombus as well (Fig. I). A fish-mouth incision was made around the orifice of the greater saphenous vein and the tumour was resected together with the thrombus adhering to it and protruding into the femoral vein. The vein was closed with interrupted Prolene 5/0. sutures Arteriovenous fistula was not cre- 


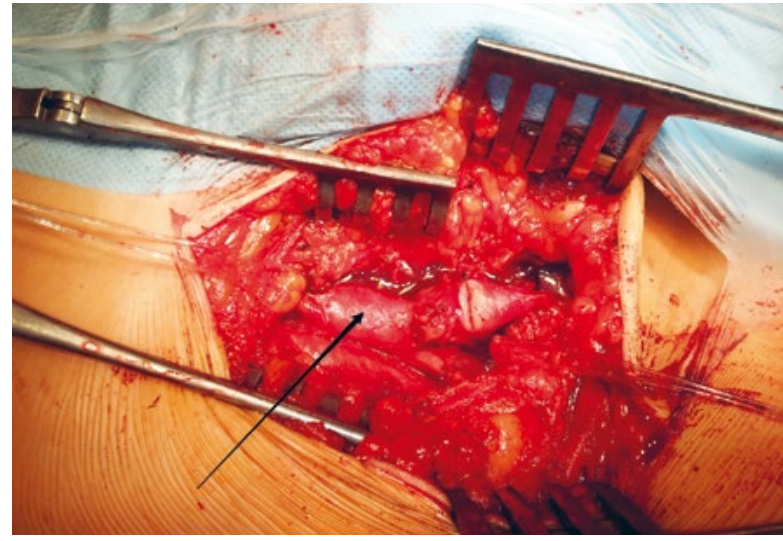

Figure I. The cross section of the dilated proximal part of greater saphenous vein containing the tumour. The arrow points common femoral vein

ated since the thrombus originated from saphenous vein only. The whole specimen was submitted for pathologic evaluation. Grossly it consisted of a firm whitish mass measuring $3.1 \times 1.7 \times 1.2 \mathrm{~cm}$ and it proved to be an atypical smooth muscle tumour leiomyoma symplasticum. There was no mitotic activity or areas of necrosis in the tumour. It was classified as a benign lesion. The patient recovered spontaneously and was discharged home. She was followed up and 6 months later color duplex Doppler imaging showed a mild $50 \%$ stenosis of the femoral vein probably at the line of stitches (Fig. 2). Mild oedema of the leg was present.

\section{Discussion}

Tumours arising from venous wall are rare. They may originate from smooth muscles of the venous wall like leiomyosarcomas and leiomyomas or from endothelial cells like hemangioendotheliomas [I, 2]. Smooth muscle tumours tend to appear in the central parts of the body and their presence in peripheral vessels is certain rarity. Leiomyomas of the uterus may spread into veins and those arising in the inferior vena cava can present similar tendency [ $I]$. Malignant potential of leiomyomas depends on their size, mitotic activity and necrosis areas. It is presumed that tumours smaller than $5 \mathrm{~cm}$ tend to be benign. The tumour which we present was small and easy to overlook in imaging studies. Some authors state that mitotic rate is the only reliable predictor of malignancy. Four and more mitotic figures per 10 high power fields are significant [ [, 3]. In presented tumour the mitotic activity was none. Also necrosis in the tumour was not observed so it was classified as benign lesion.

In our case the solitary thrombus in femoral vein was the main abnormality found in color duplex scanning and was assumed to be a segmental thrombosis of that vein.

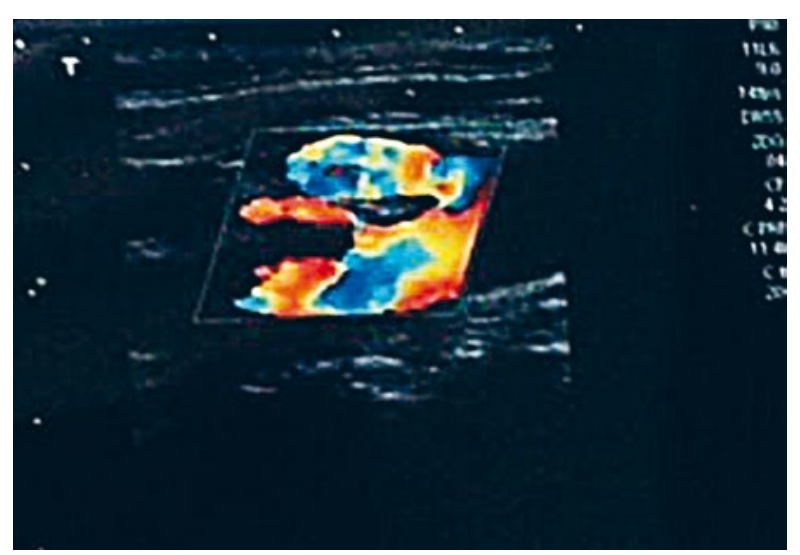

Figure 2. Femoral veins with postthrombotic septa (black areas) and impaired blood flow

The tumour was not visible. The risk of pulmonary embolism was an indication for surgery. Thrombosis of the distal part of the affected vein may develop whereas the thrombosis of the proximal part is unusual [4]. Smooth muscle tumour can sometimes mimic other groin pathologies [4]. Thrombus protruding into another vein is frequently connected with thrombosis of renal and adrenal vein due to malignancies in these organs [5].

Similar long thrombus originating from the orifice of the greater saphenous vein is very rare and should increase the spectrum of pathologies taken into account during the evaluation.

Small venous tumours especially non-palpable ones are difficult to detect. CT scan and MRI are thought to be gold standard for diagnosis of soft tissue tumours and venous tumours as well. MRI seems to be more effective although both techniques provide visualization of the tumour and confirm its venous origin [2-4].

\section{Conclusions}

I. Segmental thrombosis of large vein can be connected with an occult underlying pathology.

2. Color duplex Doppler alone does not allow proper evaluation of the problem especially if the tumour is not palpable and located inside or close to the blood vessel.

3. MRI seems to be very useful in such cases and allows to avoid making diagnosis only during surgery.

\section{References}

I. Grimer RJ, Armstrong GR. Intra-vascular leiomyoma of the popliteal vein. Postgrad Med J. 1988; 64(749): 247-248, indexed in Pubmed: 3174546.

2. Le Minh T, Cazaban D, Michaud J, et al. Great saphenous vein leiomyosarcoma: a rare malignant tumor of the extremity-two case reports. Ann Vasc Surg. 2004; 18(2): 234-236, doi: 10.1007/s 10016-003-0090-2, indexed in Pubmed: 15253262. 
3. Reix T, Sevestre H, Sevestri-Pietri MA, et al. Primary malignant tumors of the venous system in the lower extremities. Ann Vasc Surg. 1998; 12(6): 589-596, doi: 10.1007/s100169900205, indexed in Pubmed: 9841691.

4. Dellaportas D, Kotsis T, Carvounis E, et al. Leiomyoma of the greater saphenous vein mimicking inguinal lymphadenopathy.
Case Rep Surg. 2013; 2013: 23739I, doi: 10.1 155/2013/23739I, indexed in Pubmed: 24363945.

5. Gagné-Loranger M, Lacombe L, Pouliot F, et al. Renal cell carcinoma with thrombus extending to the hepatic veins or right atrium: operative strategies based on $4 \mathrm{I}$ consecutive patients. Eur J Cardiothorac Surg. 2016; 50(2): 317-321, doi: 10.1093/ ejcts/ezw023, indexed in Pubmed: $\underline{27016196 .}$ 